\title{
Osservazioni della Cometa 1884 III (Wolf)
}

fatte nel R. Osservatorio di Brera in Milano (Refr. 8 pollicidi Merz)

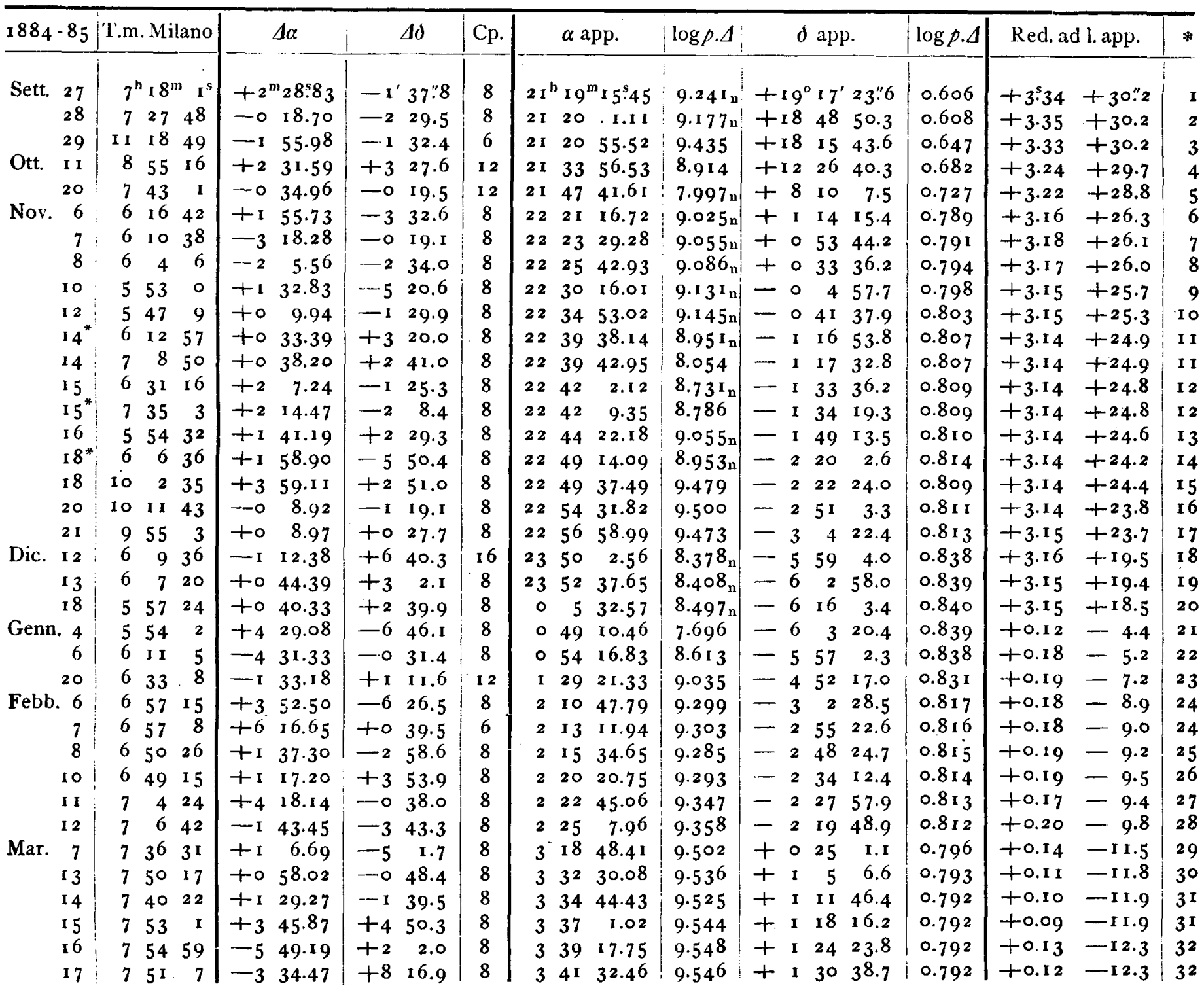

Posizionimediedelle stelle di comparazione.

\begin{tabular}{|c|c|c|c|}
\hline * & $\alpha$ I 884.0 & $\delta_{I} 8884.0$ & Autorità \\
\hline I & $21^{h} \times 6^{m} 43^{s} \cdot 28$ & $+19^{\circ} 18^{\prime} 3 x^{\prime \prime} 2$ & I Pegasi. Berl. Jahrb. \\
\hline 2 & $21 \quad 20 \quad 16.46$ & +185049.6 & BB. VI $+18^{\circ} 4789$ \\
\hline 3 & 212248.17 & +181645.8 & Rü. 9198 \\
\hline 4 & $\begin{array}{lll}21 & 31 & 21.70\end{array}$ & +122243.0 & BB. $V I+12^{\circ}{ }^{6} 650$ \\
\hline 5 & $\begin{array}{lll}21 & 48 & 13.35\end{array}$ & $+895^{8.2}$ & $W_{1} 1077$ e 1095 \\
\hline 6 & $\begin{array}{lll}22 & 19 & 17.83\end{array}$ & +11721.7 & $W_{1} 375$ \\
\hline 7 & $22 \quad 2644.38$ & $+\circ 5337.2$ & $W_{1} 525$ \\
\hline 8 & $22 \quad 27 \quad 45.32$ & $+\circ 3544.2$ & Schjell. 9225 \\
\hline 9 & $\begin{array}{lll}22 & 28 & 40.03\end{array}$ & 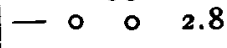 & Sant $_{5}$ I 281 \\
\hline 10 & $22 \quad 34 \quad 39.93$ & $-\circ 4033.3$ & Schjell. 9277 \\
\hline
\end{tabular}

\begin{tabular}{|c|c|c|c|}
\hline$*$ & a. 1884.0 & $\delta \mathrm{I} 884.0$ & Autorità \\
\hline $1 \mathbf{I}$ & $22^{\mathrm{h}} 39^{\mathrm{m}} \quad$ I.6 I & $-1^{\circ} 20^{\circ} 38.7$ & Schjell. 9307 \\
\hline I 2 & $\begin{array}{lll}22 & 39 & 51.74\end{array}$ & -13235.7 & Glasg. 5936 \\
\hline 13 & $\begin{array}{lll}22 & 42 & 37.85\end{array}$ & - I 527.4 & Sant $_{5} 1298$ \\
\hline 14 & $2247 \quad 12.05$ & -21436.4 & Sant $_{5} \times 3^{\circ} 3$ \\
\hline I 5 & $22 \quad 45 \quad 35.24$ & - 22539.4 & Glasg. $597^{\circ}$ \\
\hline 16 & 225437.60 & -2508.0 & $W_{1}$ I I I 10 \\
\hline 17 & $22 \quad 5^{6} \quad 4^{6.87}$ & -3513.8 & $\operatorname{Lam}_{3} 4700$ \\
\hline 18 & 235111.78 & -663.8 & $W_{1}$ I01 6 \\
\hline 19 & $255^{1} \quad 50.11$ & 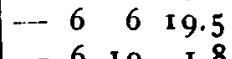 & $W_{1} 1029$ \\
\hline
\end{tabular}




\begin{tabular}{|c|c|c|c|c|c|c|c|}
\hline * & $\alpha 1885.0$ & $d_{I} 885.0$ & Autorità & $*$ & $\alpha 1885.0$ & $\delta_{I} 885.0$ & Autorità \\
\hline 2 I & $0^{\mathrm{h}} 44^{\mathrm{m}} 4^{15} .26$ & $-5^{\circ} 5^{6^{\prime}} 29^{\prime \prime} 9$ & Sant $_{2}$ Zona III & 27 & $2^{\mathrm{h}} 18^{\mathrm{m}} 26^{\mathrm{s}} .75$ & $-2^{\circ} 26^{\prime}$ I $0.0^{\prime \prime}$ & $W_{1} 266$ \\
\hline 22 & ○ 5847.98 & $-5 \cdot 56 \quad 25 \cdot 7$ & $W_{1} 1003$ & 28 & $2265 \mathrm{I} .2 \mathrm{I}$ & $\begin{array}{lll}-2 & \text { I } 5 & 55.8\end{array}$ & Sant $_{5}$ I 99 \\
\hline 23 & I $30.54 .3^{2}$ & $-4 \quad 5321.4$ & Schjell. 485 & 29 & $31741.5^{8}$ & to 3014.3 & Schjell. 989 \\
\hline 24 & 2655.11 & -25553.1 & Glasg. 485 & 30 & $\begin{array}{lll}3 & 31 & 31.95\end{array}$ & +166.8 & $W_{1} 549$ \\
\hline 25 & 21357.16 & $\begin{array}{lll}-2 & 45 & 16.9\end{array}$ & $W_{1} 187$ & $3 I$ & $333 \quad 15.06$ & +1 1337.8 & BB. VI $+1^{\circ} 640$ \\
\hline 26 & $2193.3^{6}$ & $\begin{array}{lll}--2 & 37 & 56.8\end{array}$ & Struve P.M. 234 & $3^{2}$ & $\begin{array}{lll}3 & 45 & 6.81\end{array}$ & +12234.1 & Glasg. 902 \\
\hline
\end{tabular}

Le tre osservazioni del I4, is e I 8 Novembre, delle quali la data è segnata coll' asterisco, sono state fatte dal Dr. Porro. Le osservazioni del Febbrajo e del Marzo sono state in generale alquanto difficili, quelle del 13 e del 14 Marzo sono segnate come meno sicure delle altre.

Reale Osservatorio di Brera in Milano, 1885 Giugno 15.

G. V. Schiaparelli.

\section{Vermischte Nachrichten.}

Photographie von Himmelskarten in der Milchstras se. Ueber die neuesten sehr bemerkenswerthen Erfolge der Herren Paul und Prosper Henry in der Himmelsphotographie macht der Director der Pariser Sternwarte, Contreadmiral Mouchez, in der Sitzung der Akademie vom I 5. Juni d. J. folgende interessante Mittheilung:

- Le nouvel appareil de photographie céleste de MM. Henry est entièrement terminé depuis quelques jours, et nous avons pu profiter du beau temps actuel pour obtenir de magnifiques photographies dans la Voie lactee.

Dans le cliché présenté aujourd'hui à l'Académie, on peut compter 5000 étoiles environ de la $6 \mathrm{e}$ à la $15^{\mathrm{e}} \mathrm{gran}$ deur, comprises dans une étendue de $2^{\circ}$ I $5^{\prime}$ en ascension droite et $3^{\circ}$ en déclinaison.

Pour répondre à la juste objection de M. Fizeau sur la possibilité de confondre des accidents de la plaque avec des étoiles, il a été fait trois poses successives d'une heure chacune en faisant chaque fois mouvoir la lunette de $5^{\prime \prime}$. Chaque étoile est donc représentée trois fois, formant un petit triangle de $5^{\prime \prime}$ de côté.

Pendant trois heures consécutives, l'astronome a dû conserver l'oeil et la main à la lunette pour la maintenir rigoureusement fixée sur ce même point du ciel.

Avec un microscope grossissant vingt ou trente fois, tous les détails de cette photographie ressortent avec une grande netteté.

Ce résultat très remarquable fait disparaître les derniers doutes qu'on pouvait conserver sur la pussibilité d'entreprendre aujourd'hui la Carte de toute la voûte céleste, en y introduisant à très peu prés toutes les étoiles visibles avec les plus forts instruments. A l'aide de semblables clichés, l'astronome pourra continuer à explorer et à étudier le ciel dans son cabinet, avec un simple microscope, quand le temps couvert ne lui permettra pas d'observer.

Pour représenter les $41000^{\circ}$ superficiels de. la voûte céleste, il faudrait 6000 clichés semblables formant I 500 de nos Cartes écliptiques.

En admettant que six ou huit observatoires bitn situés dans les deux hémisphères s'entendissent pour entreprendre ce travail, et que chacun d'eux fit 150 ou 200 clichés par an, une Carte complète du ciel, contenant plus de vingt millions d'étoiles jusqu'à la $14^{\mathrm{e}}$ et la $15^{\mathrm{e}}$ grandeur, pourrait être exécutée en moins de cinq à six ans.

Ce serait certainement l'oeuvre astronomique la plus considérable et la plus importante qui aurait jamais été exécutée, et qui léguerait aux astronomes de l'avenir un état très exact du Ciel à la fin du XIX" siècle, sans erreur ni omission possibles.

L'observatoire de Paris est prêt à entreprendre sa part de ce travail avec le grand appareil photographique de 0.34 d'ouverture, que viennent de construire MM. Henry et Gautier. Nous allons l'utiliser, en attendant, à continuer la Carte écliptique. «

\section{In halt:}

Zu Nr. 2671. Chas. H. Kummell. Un the determination of the shortest distance between two points un a spheroid, 97 . - Aufforderung betreffend Beobachtungen des Cometen 1884 III (Wolf). ro7. - G,V. Schiaparelli. Osservazioni della Cometa 1884 III (Wolf). rog. Vermischte Nachrichten. I I I. 